\title{
Construction of the Diagnosis and Treatment Process of Dermatosis Based on Data-Driven Approach
}

\author{
XingLiang Qi \\ Shandong University of Traditional \\ Chinese Medicine \\ Institute of Chinese Medical History \\ and Literature \\ Jinan, China
}

\author{
Yang Zhou \\ Shandong University of Traditional \\ Chinese Medicine \\ Institute of Chinese Medical History \\ and Literature \\ Jinan, China \\ ZhenGuo Wang \\ Shandong University of Traditional \\ Chinese Medicine \\ Institute of Chinese Medical History \\ and Literature \\ Jinan, China \\ zhenguow@126.com
}

\author{
XianJun Fu \\ Shandong University of Traditional \\ Chinese Medicine \\ Institute of Chinese Medical History \\ and Literature \\ Jinan, China
}

\begin{abstract}
In this study, we selected the dermatology data, constructed a dictionary of dermatology semantic classification, and established a corpus of dermatology literature. Based on the context calculation model we took the case of erythema skin disease as the example, identified the semantic meaning of the dermatology data, and formed the concept attribute of dermatology knowledge, constructed the dermatology ontology. We established a priori database and verified each other with the running data to correct the data error and improve the data definition. In this study, we used the natural language to describe the characteristic information of skin diseases, and carried out the semantic searching. Finally, we constructed the mathematical model of the diagnosis and treatment of dermatology based on the corpus, the ontology and the priori database. The purpose is to supply the gap of unbalance of doctors' personal level of the dermatosis diagnosis and treatment, improve the level and accuracy of clinical diagnosis and treatment of dermatosis, and to achieve effective drive of medical literature data for clinical diagnosis and treatment.
\end{abstract}

Permission to make digital or hard copies of all or part of this work for personal or classroom use is granted without fee provided that copies are not made or distributed for profit or commercial advantage and that copies bear this notice and the full citation on the first page. Copyrights for components of this work owned by others than ACM must be honored. Abstracting with credit is permitted. To copy otherwise, or republish, to post on servers or to redistribute to lists, requires prior specific permission and/or a fee. Request permissions from permissions@acm.org.

WI '17, August 23-26, 2017, Leipzig, Germany

(c) 2017 Association for Computing Machinery.

ACM ISBN 978-1-4503-4951-2/17/08...\$15.00

http://dx.doi.org/10.1145/3106426.3109047

\section{KEYWORDS}

Dermatology, Clinical Diagnosis and Treatment, Natural Language Processing, Corpus, Ontology

\section{ACM Reference format:}

X. Qi, Y. Zhou, X. Fu and Z. Wang. 2017. Construction of the Diagnosis and Treatment Process of Dermatosis Based on Data-Driven Approach. In Proceedings of WI '17, Leipzig, Germany, August 23-26, 2017, 4 pages. http://dx.doi.org/10.1145/3106426.3109047

\section{INTRODUCTION}

There are more than 3000 kinds of skin diseases mentioned in China Clinical Dermatology [1]. In International Classification and Nomenclature of The Skin Disease there are 8808 names of skin diseases in Chinese and 8940 in English [2]. Such a large variety of diseases causes clinical diagnosis and treatment difficulties, and so misdiagnosis or missed diagnosis often occurs [3]. Misdiagnosis may happen frequently even in terms of clinically common diseases. One of the key causes of these diagnosis errors is that the complexity of dermatology and of the dermatology literature is too much difficult for doctors. One of the most important bases for the diagnosis of skin disease is the configuration of the skin lesions, and thus the most important way to have a total knowledge of skin disease is to watch the skin lesions and make and a complete diagnosis and treatment process. Due to the differences in the level of hospitals and in the number of patients, the total amount of skin diseases found in each region and hospital is much different which therefore leads to different levels of clinical diagnosis and treatment. In order to improve doctors' work efficiency, automated tools are 
desperately needed to help doctors' to find the information they need in the massive biomedical literature. Therefore, we have constructed a data-driven dermatology clinical diagnosis and treatment process to help doctors improve the efficiency and accuracy of diagnosis and treatment.

Taking erythema skin diseases as the research object, we selected the dermatology corpus, and built a dictionary of dermatology semantic classification and a corpus of dermatology literature. By a ontology for dermatology, we completed the construction of diagnosis and treatment process of erythema skin disease.

Erythema is one of the most common symptoms of skin diseases and is mentioned in the vast majority of dermatology literature. Thus, taking erythema symptoms as the research object is fairly helpful to the clinical diagnosis and treatment.

\section{DATA SELECTION AND PROCESSING}

\subsection{Selection criteria of corpus data}

(1) Strong authority, great guiding significance

(2) Full content, wide coverage

(3) Uniform standards, less ambiguity

The corpus data we selected is based on the China Clinical Dermatology (Zhao Bian, Jiangsu Science Press, January 2010, first edition). The book is updated and modified from the third edition of Clinical Dermatology which is the most popular professional book among dermatologists and is recognized as the most authoritative reference book for dermatologists.

\subsection{Construction of Dermatology Semantic Dictionary}

In order to realize the semantic filtering in the literature knowledge discovery, this dictionary uses the two-dimensional table files of the relational database to describe the grammatical attributes of words. By classifying different attributes of each word, this dictionary has established a word processing system for information processing, and created a machine dictionary targeted at the specialized field and direct services for information processing [4].

\subsection{Construction of Dermatology Literature Annotation Corpus}

We used the tool "Chinese Word Segmentation and Part-ofspeech Tagging" developed by Peking University [5], to generate a primary corpus. Then, we constructed a professional corpus of dermatology by using the segmentation dictionary of dermatology to mark the label of the words.

\subsection{Construction of Dermatology Ontology}

We completed the construction of dermatology ontology by making top-level concepts, the attribute description system and the analysis of samples.

Top-level concept of erythema: disease name, summary, etiology and pathogenesis, clinical manifestations, diagnosis,

dermatopathology, laboratory tests, prevention, treatment, etc. Table 1.

Attribute description of erythema: color, category, accompanying symptoms, causes, incidence, prevalence, age of onset, symptom distribution, etc. Each attribute can be described in several ways. For example, the description of color: red, purple, rose-red, pink; the description of category: edematous erythema, angioedema, telangiectasia, erythroderma, erythema multiforme alike etc. Table 1.

Table 1: Top-level concept and attribute description of erythema

\begin{tabular}{ll}
\hline Top-level concept of erythema & \\
\hline disease name, outline, etiology, pathogenesis, clinical \\
manifestations, diagnosis, histopathology, laboratory examination, \\
prevention, treatment
\end{tabular}


Construction of the Diagnosis and Treatment Process of Dermatosis Based on Data-Driven Approach

Processing of characteristic symptoms and manifestations: Some symptoms and manifestations can be served as the characteristics or features to the skin diseases. We collected these characteristics into a database which is helpful to the diagnosis of skin diseases. These characteristic are in line with specific diagnoses. For example, a Koplik patch will help to give a diagnosis of the measles; a "slapped cheek" erythema will help to give a diagnosis of erythema infectiosum; a symmetrical fusion of purplish red spots that occur frequently in the face will help to give a diagnosis of dermatomyositis. There are 60 items of these characteristics-diagnosis rules.

The setting of key nodes: the key nodes are the symptoms or disease characteristics which have important meaning or key basis in the diagnosis. The addition of nodes makes the diagnosis process more concise, more accurate and efficient; it can also play a role in the clinical interrogation. The setting of key nodes should not be too much, so as to ensure the simplicity of clinical diagnosis. In this study, we set up 6 nodes, which were accompanied by symptoms, typical etiology, infection or epidemic, erythema type, color and shape, distribution.

\subsection{Construction of the Priori Database}

Validation settings: Dermatologist have screened out all the erythematous skin diseases, which were used as the prior data base, and verified with the running data, corrected the error, in order to optimize the operating rules.

The feedback of the data analysis: Compared with the running data, the data definition can be improved by correcting the data result error. Under the premise of improving the accuracy of data, this feedback can reduce the ambiguity of the semantic dictionary, and correct the deviation in the construction of ontology, can enhance the extraction of characteristic rules, and improve the key node settings and the accuracy of the results.

\section{CONSTRUCTION OF DIAGNOSIS AND TREATMENT PROCESS}

It is consistent with the clinical diagnosis and treatment process that starts with symptoms. The patient will focus on the evolution of symptoms in the course of the development of the disease. Here to explain taken erythema symptoms as an example. The initial symptom of many diseases is erythema, such as rubella, contact dermatitis, lupus erythematosus, etc. Erythema can also occur in the development of some diseases, such as congelatio, the first symptoms appear pale skin, cold, pain and numbness, and then showed erythema, edema and other symptoms. The patient may be at any stage of the disease, therefore, whether it is not the first symptoms, erythema can be used as the main symptoms or accompany symptoms to see a doctor. In this study, the diagnosis and treatment process can be effective in the treatment of erythema symptoms of skin diseases including all the symptoms of erythema. The flow chart of the diagnosis and treatment process is shown in Figure 1.

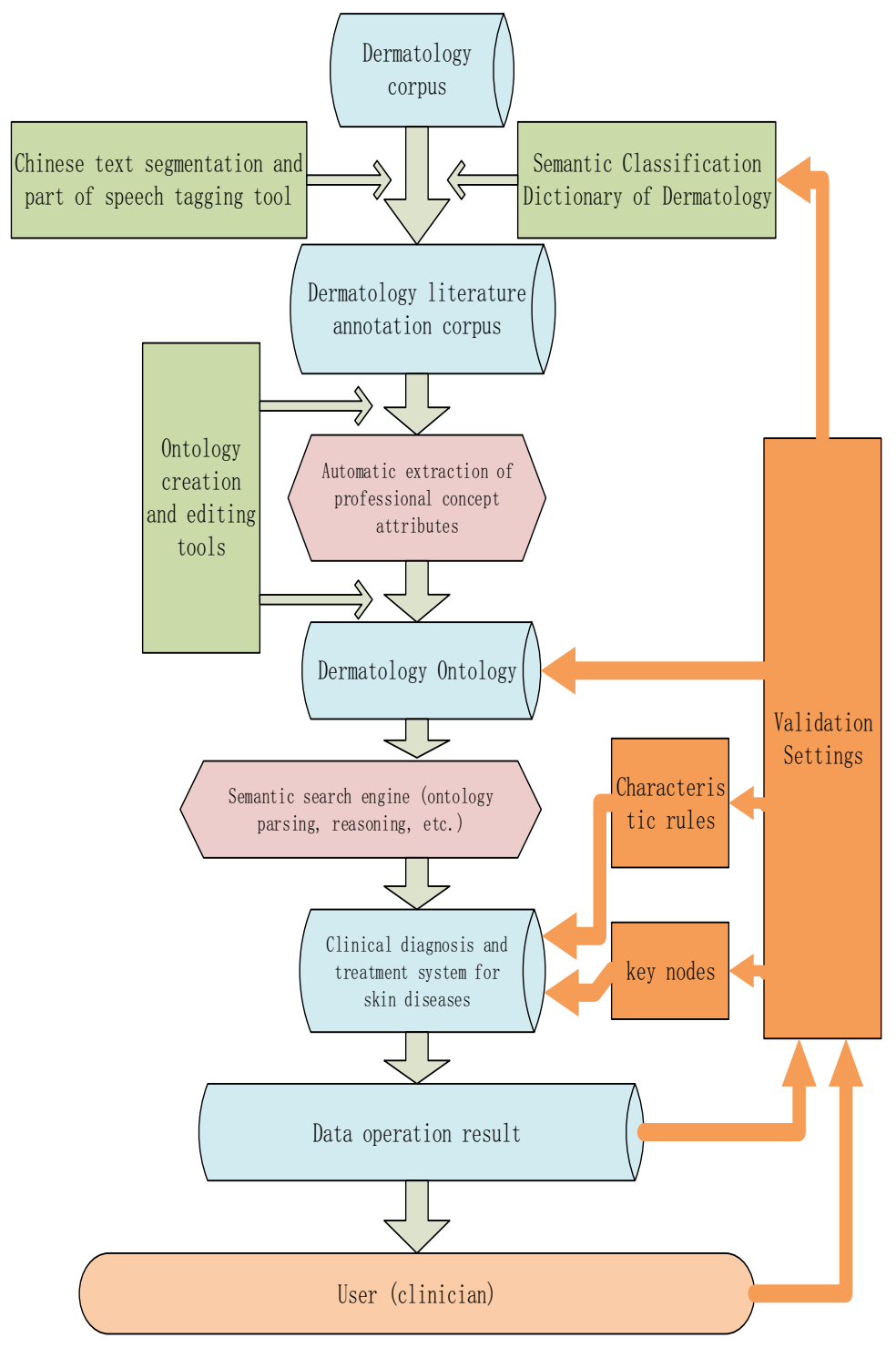

Figure 1: The flow chart of the diagnosis and treatment process

\section{CONCLUSIONS}

In this paper, using the technology and method of natural language processing, we selected and built the dermatology corpus, constructed the dermatology ontology, and established a priori database to provide knowledge and data correction in the very step of the diagnosis and treatment process. All these efforts we made can help doctors obtain information according to requirement of the dermatosis, mutual relationships and potential links of a variety of information in order to reduce the subjective bias and can improve the level and accuracy of doctors' diagnosis and treatment of dermatosis, and can achieve 
effective drive of medical literature data for clinical diagnosis and treatment.

The priori data were screened and identified by experienced dermatology experts and the database will be constantly modified as the system is applied to the clinical process. The diagnosis and treatment system has been applied by dermatology doctors and has received good feedback. Some of the following problems need to be further improved: 1 . How to further improve the integrity and accuracy of the running data; 2. How to reflect the clinical complexity of skin diseases. In the next step, we will use the continuous improvement of the priori database to control and improve all aspects of the system, so that the construction ideas and methods of this system are more mature and complete. As more skin disease data is used and more skin symptoms are included, the clinical complexity of skin diseases will gradually be represented accurately and comprehensively.

\section{ACKNOWLEDGMENTS}

This work is partially supported by 2 projects: 1 . The key research project of ShanDong province (No.2016GSF202028 and No.2016CYJS08A01-1); 2. Special post doctoral innovation program of ShanDong Province (2016). The authors also gratefully acknowledge the helpful comments and suggestions of the reviewers, which have improved the presentation.

\section{REFERENCES}

[1] Zhao Bian. 2010. China Clinical Dermatology [M]. Jiangsu Science and Technology Press.

[2] Ma Zhenyou, Shi Xin, Liu Aimin and Dai Changlong. 2007. International Classification And Nomenclature of The Skin Disease [M]. Publishing House of Ancient Chinese Medical Books.

[3] Liang Zuohui. 2016. Analysis of the causes of misdiagnosis in 327 cases of herpes zoster. fournal of Dermatology and Venereology 4, (38), 134-135.

[4] Xingliang Qi, Chin Wei, Ting Yuan, Zhenguo Wang and Yang Zhou. 2016. Research on the diagnosis and treatment process of dermatosis based on datadriven approach. ICIC Express Letters Part B: Applications, Vol. 7, No. 11, 23672372.

[5] Y. Liu, Z. Zhang and Y. Huang. 2016. Research and development of semantic annotation platform for scientific literature. ICIC Express Letters, Vol. 10, No. 7, 1787-1794. 